\title{
Spécialisations industrielles, structures sociales, activités financières et intégration économique internationale au XIXe siècle : le cas de la Grande- Bretagne et de la France
}

Industrial specialisation, social structures, financial activities and international economic integration in the $19^{\text {th }}$-century: the cases of Great Britain and France.

\section{Patrick Verley}

\section{OpenEdition}

\section{Journals}

Édition électronique

URL : http://journals.openedition.org/rh19/361

DOI : $10.4000 /$ rh 19.361

ISSN : $1777-5329$

Éditeur

La Société de 1848

Édition imprimée

Date de publication : 1 décembre 2001

Pagination : 47-71

ISSN : 1265-1354

Référence électronique

Patrick Verley, "Spécialisations industrielles, structures sociales, activités financières et intégration économique internationale au XIXe siècle : le cas de la Grande-Bretagne et de la France ", Revue d'histoire du XIXe siècle [En ligne], 23 | 2001, mis en ligne le 19 mai 2009, consulté le 03 mai 2019. URL http://journals.openedition.org/rh19/361 ; DOI : 10.4000/rh19.361

Ce document a été généré automatiquement le 3 mai 2019.

Tous droits réservés 


\section{Spécialisations industrielles, structures sociales, activités financières et intégration économique internationale au XIXe siècle : le cas de la Grande-Bretagne et de la France}

Industrial specialisation, social structures, financial activities and international economic integration in the $19^{\text {th }}$-century: the cases of Great Britain and France.

\section{Patrick Verley}

1 La période qui commence aux lendemains de 1815 et se termine en 1914 est caractérisée par une relation plutôt pacifique entre les principales puissances. Les guerres sont restées courtes et ponctuelles et les principales d'entre elles furent liées à la dynamique de réalisation d'espaces nationaux tardivement unifiés, comme la guerre austro-prussienne, celle des duchés danois et la guerre franco-prussienne de 1870-1871. Il n'y avait derrière ces conflits aucune motivation économique, ce qui n'était pas le cas dans ce XVIII ${ }^{\mathrm{e}}$ siècle conflictuel qui, au terme des French Wars, se termina par la défaite française en 1815. Ce ne sera pas toujours le cas dans un $\mathrm{XX}^{\mathrm{e}}$ siècle où les conflits furent, dans le monde, très prégnants. Certes, dans les années 1880-1890 s'ouvrit une période plus conflictuelle dans les relations internationales. La montée des conflits douaniers révéla une apparition, décalée par rapport au politique, de formes de nationalismes économiques, qui incitaient les contemporains à utiliser un vocabulaire belliqueux pour décrire, par exemple, les tensions commerciales entre la France et l'Italie ou la France et la Suisse. Mais, même durant cette période, les conflits politiques et la formation des grands systèmes d'alliances restèrent très indépendants des intérêts économiques. 
2 Le XIX ${ }^{e}$ siècle consacra une certaine dépolitisation de l'économie dans la mesure où les conflits économiques furent réglés par des négociations économiques et non par l'usage de la force politique. Cela fut possible parce qu'il s'était instauré une division du travail simple mais efficace entre les pays en industrialisation, s'accordant aux avantages comparatifs en matière de structure de prix, de structure organisationnelle et d'héritages en savoir faire. Cette division du travail attribuait au milieu du siècle à chacun des pays en industrialisation une place bien distincte. Ce système commercial, c'est-à-dire cet ensemble de flux commerciaux hiérarchisés, s'appuya sur un multilatéralisme qui facilitait les paiements et engendrait un ensemble de flux de capitaux à court terme assurant les compensations dans le cadre d'une division du travail entre centres de paiements. Cette relative harmonie des années 1840-1860 fut ensuite progressivement perturbée par le développement de l'industrialisation qui modifia les avantages comparatifs relatifs et, partant, la structure des importations et exportations des différents pays, avec un effet d'entrée sur le marché international de "nouveaux pays industriels".

3 Le mode d'insertion de l'économie française dans l'économie internationale --aussi bien pour les flux de marchandises que pour les flux de capitaux-- se modifia en conséquence entre le milieu du siècle et le début $\mathrm{du} \mathrm{XX}^{\mathrm{e}}$ siècle où s'est désormais effectuée une recomposition du système de l'économie internationale avec de nouveaux flux de biens, de services et de paiements que les travaux classiques et pionniers de Samuel Saul ${ }^{1}$ ont décrits, il y a déjà quarante ans.

\section{La genèse d'un système fondé sur une division internationale du travail généralisée}

$4 \mathrm{Au} \mathrm{XVIII}{ }^{\mathrm{e}}$ siècle la division internationale du travail n'était que très limitée entre les économies européennes au niveau du commerce spécial ${ }^{2}$. Au niveau du commerce général, elle opposait les pays réexportateurs de produits tropicaux et les autres. Elle était rudimentaire au niveau mondial, opposant les économies coloniales fournissant des produits tropicaux et les économies des pays avancés européens vendant tous, pour l'essentiel, les mêmes produits, fabriqués dans des structures proto-industrielles avec les mêmes techniques et donc la même productivité. En effet s'il y avait sur des produits comme les soieries une division du travail déjà assez fine selon la qualité entre les producteurs lyonnais, italiens, hollandais ou anglais ${ }^{3}$, la concurrence était plus directe entre les grands centres drapiers et encore plus pour la toilerie. Sur les quelques marchés extra européens ouverts où le protectionnisme général ne verrouillait pas les échanges, la concurrence était donc féroce et les états qui appliquaient des politiques industrialistes pour créer du travail et éviter les explosions sociales, soutenaient par l'action politique leurs producteurs. Du fait des rivalités coloniales et industrielles, une partie non négligeable du coût de fonctionnement du commerce international était ainsi le fait des dépenses militaires des États. Une fois les Provinces-Unies mises hors course, le conflit se focalisa sur un affrontement entre deux blocs, le bloc hispano-français et le bloc lusitanobritannique. Des ébauches de systèmes de paiements multilatéraux ou plus exactement de circulation des métaux précieux entre les pays de production et les pays qui se trouvaient en position de thésaurisation finale, existaient donc autour de chacun des deux blocs commerciaux. Grâce à ces systèmes partiels de paiement, comme celui que S. B. Saul a reconstruit autour de la Grande-Bretagne du milieu du XVIII ${ }^{e}$ siècle ${ }^{4}$, les métaux des 
colonies ibériques permettaient in fine de payer les déficits commerciaux sur l'ExtrêmeOrient et sur les pays de la Baltique, au terme parfois de détours complexes : par exemple par l'Amérique latine, les colonies antillaises et les colonies nord-américaines. Des circuits comparables qu'il faudrait décrire existaient sûrement autour de l'économie française. La bipolarisation qui était la conséquence du rapport des métropoles aux économies coloniales faisait obstacle au développement d'un système économique international.

$5 \quad \mathrm{Au} \mathrm{XIX}^{\mathrm{e}}$ siècle au contraire, le fait colonial n'imposait plus son empreinte à l'économie internationale et une division du travail complexe s'était désormais aussi mise en place entre exportateurs de produits manufacturés. Elle se situait à l'intérieur de la production manufacturière par la diversification des produits à deux niveaux. Le premier niveau était constitué par les stades successifs d'élaboration des produits qui se multipliaient et donnaient lieu à des activités productives autonomes. Le processus d'industrialisation développait ainsi un nombre croissant de produits intermédiaires, dont les capacités de production ne progressaient pas à la même vitesse. La diversification des produits s'opérait aussi par leur segmentation en de nombreuses qualités. Cette évolution fut surtout le fait des produits de consommation, comme les tissus, mais elle s'appliquait également aux matières premières et aux demi-produits comme les filés, les fers ou les houilles. Cette évolution a été voulue par les producteurs pour mieux s'adapter à la demande, ainsi que pour développer cette demande, hâter l'obsolescence des produits et s'assurer des marchés captifs. Pour ce qui est des tissus, la variété des produits proposés à la clientèle s'accrut beaucoup à partir de 1820 avec une exploitation systématique de l'effet de mode pour ce que l'on appelait les "tissus de nouveauté". Ces stratégies commerciales d'innovation de produits et de segmentation de marchés qui furent rapidement généralisées pour l'ensemble des biens de consommation concernaient également les produits intermédiaires. Si la variété des tissus nécessitait une grande variété de filés, la multiplication des usages de demi-produits comme les produits sidérurgiques ou même la houille avait pour conséquence une multiplication et une spécialisation des qualités dont les prix pouvaient fortement varier : d'un multiplicateur considérable entre le fer ordinaire et l'acier pour ressorts de montres. Cette segmentation par la qualité n'était pas seulement le reflet passif de la demande des industriels usagers mais aussi une manière pour les producteurs de valoriser au mieux leur production ${ }^{5}$. Du fait de cette complexification de la nomenclature des produits dont le foisonnement des nomenclatures douanières ${ }^{6}$ rendait compte, la division du travail entre pays se faisait de plus en plus transversalement aux nomenclatures principales, par le développement d'un commerce intra-branches.

\section{Le système commercial du milieu du XIX ${ }^{e}$ siècle}

6 Au milieu du XIXe siècle, la structure du commerce international était simple, car seuls quatre pays exportaient une majorité de produits industriels et importaient des produits alimentaires et des matières premières : la Grande-Bretagne, la France, la Belgique et la Suisse. Les échanges de ces quatre pays pouvaient par ailleurs être réduits dans un schéma très simplifié à deux polarités, le commerce britannique et le commerce français pour deux raisons ${ }^{7}$. D'abord en poids, même si leurs taux d'ouverture étaient beaucoup plus élevés, la Belgique et la Suisse ne comptaient qu'assez peu dans le commerce international. Ensuite les avantages comparatifs belges ressemblaient à ceux de 
l'Angleterre, ceux de la Suisse étaient proches des Français. Quant aux autres pays, ils étaient tous fournisseurs de produits agricoles et miniers et acheteurs de produits manufacturés, même ceux qui étaient à un niveau d'industrialisation proche des pays les plus avancés comme c'était le cas pour le Zollverein ou les États-Unis. En ne considérant que la structure des importations et des exportations dans une nomenclature opposant produits manufacturés et produits primaires, les États-Unis, les pays germaniques, méditerranéens, l'Amérique latine ou l'Asie semblaient avoir la même structure. Mais à regarder plus finement la nomenclature des produits manufacturés, on comprend que cette division élémentaire des partenaires commerciaux mondiaux en deux groupes était une situation qui n'était que transitoire. Pour ne citer que les deux partenaires les plus volumineux, le Zollverein et les États-Unis ne pouvaient qu'avoir une structure en évolution rapide. Ils étaient surtout importateurs de demi-produits dans la mesure où les entreprises en amont dans le textile et la sidérurgie ne produisaient pas encore assez pour fournir les industries aval et surtout ne produisaient pas toutes les qualités. Les Allemands devaient acheter à la Grande-Bretagne dans les années 1830 et 1840 des filés de coton surtout de grand numéro ${ }^{8}$, des fontes diverses, des rails, des machines; leurs entreprises industrielles se libérèrent progressivement de cette dépendance par rapport aux importations dans les années 1850. Les Américains ne produisaient pas non plus toute la gamme des produits de consommation, non parce qu'ils ne savaient pas le faire, mais parce que leur développement industriel n'avait pas encore pu couvrir toute la liste déjà longue des produits différents que l'industrialisation avait inventés. En effet, l'industrialisation était autant un processus d'innovation massive de produits nouveaux par spécialisation des usages et segmentation des qualités qu'un ensemble de mutations dans les procédés de fabrication des produits déjà connus. Les pays germaniques et les États-Unis ne pouvaient à terme qu'avoir une structure commerciale se rapprochant des deux premières puissances industrielles de la première moitié du XIXe siècle. Mais la structure de la balance commerciale d'un pays marquait en général un temps de retard par rapport à ses structures productives dans des modèles d'industrialisation qui étaient par substitution d'importations et non pas tirés par les exportations. La raison en était l'inertie des flux commerciaux dont l'élasticité par rapport au prix était au XIXe siècle d'autant plus faible que les marchés étaient très opaques et mal informés ${ }^{9}$, que les réseaux de commercialisation à l'étranger étaient longs à construire et que les situations acquises jouissaient d'une rente de situation. La balance commerciale de l'Empire allemand unifié allait rapidement évoluer vers une structure correspondant à celle d'un pays industriel avancé, puisque déjà en 1872 les produits manufacturés faisaient près de la moitié des exportations, pour se stabiliser à environ $65 \%$ à partir de $1890^{10}$. L'évolution allait être plus lente pour les États-Unis ${ }^{11}$ qui restèrent en fait jusqu'à 1929 des exportateurs primaires. Ce n'est néanmoins pas là un paradoxe pour le pays industriel le plus avancé, car le différentiel de productivité entre l'agriculture américaine et les agricultures européennes était sûrement beaucoup plus grand que le différentiel de productivité dans l'industrie entre les mêmes partenaires ${ }^{12}$.

7 Dans la première moitié du XIX siècle, l'importance relative des flux bilatéraux faisait que le commerce international s'ordonnait autour d'un triangle Grande-Bretagne, France et États-Unis (ces trois pays assuraient à eux seuls entre 40 et $45 \%$ des exportations mondiales dans les années 1850-1860). L'ensemble des pays allemands avait dans les années 1860 une participation presque aussi importante que la France aux échanges internationaux, mais il serait anachronique de les considérer comme une économie 
nationale homogène, alors que seule la réalité économique du Zollverein existait. Si l'on met à part les produits comme le sucre, le café, le thé, la soie ou l'indigo, les États-Unis étaient le premier fournisseur de produits primaires --coton, tabac et blé au premier chef--, et le premier acheteur de produits manufacturés dans le monde : en $185054 \%$ des exportations américaines allaient vers la Grande-Bretagne et $41 \%$ des importations en venaient.

8 La Grande-Bretagne et la France étaient les premiers acheteurs de produits primaires et les premiers fournisseurs de produits manufacturés, dans une relation non de concurrence, mais de complémentarité qui explique donc le caractère alors peu conflictuel des relations économiques internationales. M. Lévy-Leboyer avait dès 1964 évoqué une spécialisation française sur la qualitée ${ }^{13}$, c'est-à-dire une différence plus qualitative que sectorielle entre produits exportés français et britanniques. On peut démontrer que ces spécialisations sur la qualité en petites séries dans des structures déconcentrées et flexibles ou bien pour la Grande-Bretagne sur la production en grandes séries à grande productivité et donc à bas prix étaient non seulement le résultat des différences de coûts relatifs du travail, mais aussi des différences de structure sociale des marchés intérieurs des pays importateurs, et surtout --ce qui est moins évident--, des pays exportateurs ${ }^{14}$. L'analyse suédoise ${ }^{15}$ en termes de coût et rareté des facteurs de production donne certes une explication robuste de cette différence dans le système productif puisque le coût du travail non qualifié sur le continent européen était très bas. Il l'était dans la mesure où la norme salariale était fixée par rapport à la rémunération de l'ouvrier agricole dans des sociétés encore massivement rurales et agricoles, ce qui orientait vers des fabrications incorporant beaucoup de travail. En revanche le coût élevé du travail en Grande-Bretagne favorisait des structures productives très mécanisées. En outre la spécialisation au niveau des exportations ne pouvait s'affirmer que sur des produits pour lesquels il y avait une demande sociale dans les pays acheteurs, c'est-à-dire dans les pays extra-européens ouverts ou bien dans les pays européens ou aux États-Unis, ces derniers pays étant verrouillés par un protectionnisme total. Dans ces pays, les protectionnismes ne pouvaient que favoriser les complémentarités, voire les innovations de produits puisque ne pouvaient y être importés que des produits auxquels les lobbies industriels ne s'opposaient pas. Il y aurait une recherche à faire sur le rôle stimulant des obstacles érigés par le protectionnisme pour l'innovation de produits et d'organisation.

Mais les produits exportés sont en général ceux qui ont été mis au point sur le marché intérieur, ceux pour lesquels les effets cumulés d'apprentissage, acquis à l'intérieur assurent ensuite une supériorité à l'extérieur. On vend à l'extérieur ce que l'on sait bien faire pour le marché intérieur. En matière de biens de production, le pays dont l'industrie avait une avance technologique et industrielle sur les autres s'imposait nécessairement comme fournisseur. En matière de biens de consommation, les produits exportés devaient être ceux que la structure interne de la consommation --qui était conditionnée par le mode de répartition des revenus, mettait en position de force sur le marché intérieur. Cet ensemble de conditions pourrait donc sembler une quadrature du cercle si le pays acheteur et le pays vendeur avaient la même structure économique et sociale intérieure et le même degré de développement. En fait cette hypothèse est purement théorique puisque l'évolution des économies de la seconde moitié du $\mathrm{XX}^{\mathrm{e}}$ siècle révèle que plus les sociétés et les économies avancées semblent proches, plus les échanges entre elles sont intenses de par leur avancement même qui occasionne une forte différenciation des produits et une forte division du travail : les échanges intra-branches reposent sur de 
fines différences entre sociétés et économies. Au XIXe siècle, ce processus était en germe. Mais il reposait sur une forte différence au niveau des produits exportés, car il existait encore une différence importante dans les structures de consommation et de revenus des pays exportateurs de produits manufacturés. Ainsi s'est affirmée une complémentarité entre les économies britannique et française dans la première moitié du XIX ${ }^{\mathrm{e}}$ siècle.

\section{Exportations industrielles britanniques, structures de production, structures sociales}

10 Dans le système des échanges qui se mettait en place, la supériorité britannique ne pouvait être contestée. On passa d'un jeu où auparavant deux pays prenaient des initiatives en même temps pour essayer de s'assurer l'hégémonie à un jeu où il y avait un leader reconnu en fonction duquel les autres devaient ajuster leurs choix. Ces pays devaient donc trouver leurs spécialisations dans les interstices du commerce britannique, dont il faut préalablement analyser le type de spécialisation. L'évolution de la répartition du revenu national britannique que l'on peut aborder en confrontant les travaux existants sur la répartition, les évaluations des taux de croissance ${ }^{16}$ et ce que l'on sait des comportements budgétaires explique un plafonnement de la demande de produits industriels à partir de 1820. En effet, il y eut un ralentissement de la croissance des revenus des classes populaires et des classes moyennes qui avaient jusqu'alors tiré la croissance de l'industrie textile, la principale industrie de consommation, par leur demande à forte élasticité par rapport au prix et par rapport au revenu. Les gains de productivité profitaient désormais surtout aux classes aisées et moyennes. Le mode de répartition des gains de productivité qui l'emporta jusqu'aux années 1890 était en faveur des profits, des revenus des capitaux et des consommateurs du fait de la baisse des prix des produits ; il n'était pas favorable aux revenus du travail. Mais par consommateurs, il fallait surtout entendre les catégories supérieures et moyennes de la population soit celles qui profitaient aussi de la hausse des revenus autres que ceux du travail, dans la mesure où les catégories populaires n'étaient pas encore le centre de gravité des marchés de produits dans les pays en industrialisation du XIX siècle. La "révolution des consommateurs" affirmée par les historiens britanniques ${ }^{17}$ recouvrait un certain accès des classes populaires à une consommation régulière dans un marché où l'essentiel de la clientèle était constitué par les classes moyennes et supérieures. Cette "révolution" n'avait rien à voir avec la consommation de masse du XXe siècle. Ce partage des gains de productivité dans un secteur industriel déjà très important dans le produit national brut freinait la diffusion de la consommation vers le bas de la pyramide sociale, mais était en revanche favorable à la constitution d'épargne. Cela explique la surabondance de l'offre de capitaux à long terme sur les marchés de capitaux dès les années 1820 en GrandeBretagne qui trouva un débouché dans les placements et les investissements dans des pays où les marchés de capitaux étaient dans une situation inverse, comme les États-Unis ou l'Amérique latine.

11 En revanche, la demande de biens de consommation peinait à couvrir l'offre. Les industriels britanniques qui s'étaient auparavant déjà orientés vers une production de masse à bas prix pour s'adapter à un type de marché, qui comparativement aux autres pays européens était déjà socialement plus large, cherchèrent alors des marchés extérieurs pour éviter l'asphyxie. Ils avaient déjà commencé, contraints et forcés, sous le Blocus continental. Ils ne pouvaient vendre ce type de produits dans les pays qui 
commençaient à s'industrialiser, où des barrières douanières protégeaient le développement des industries textiles en train de se mécaniser. Ils se tournèrent donc vers les marchés des pays lointains où l'influence britannique était dominante, comme l'Inde dans laquelle il y avait une population à très faible pouvoir d'achat certes, ...mais très nombreuse. Le marché indien a contribué à plus d'un quart de la croissance des exportations de tissus et de la production cotonnière entre 1820 et 1855, à $66 \%$ de l'une et à $46 \%$ de l'autre entre 1856 et $1886^{18}$.

Cette nouvelle orientation vers des marchés extérieurs qui ne pouvaient acheter que des tissus grossiers à très bas prix, renforça la spécialisation des industriels britanniques dans la production de masse, confortant leur avantage comparatif sur les produits de grande série. Ils entrèrent sur le marché indien en deux temps. D'abord ils vendirent des filés aux tisserands indiens. Cela était possible car la différence de productivité entre filage à main et filature mécanique était beaucoup plus grande que celle entre tissage à bras et tissage mécanique. Dans le tissage, l'écart de productivité ne compensait pas en un premier temps l'écart de coût du travail. Mais les industriels de Manchester parvinrent bientôt à vendre aussi les tissus tout faits. Cela avait été rendu possible par un effort pour réduire les coûts, un effort important en matière technologique aboutissant au métier automatique. Ils pouvaient aussi profiter de la qualité de la main d'œuvre dont "l'autoformation" dans une région où l'activité était si concentrée ne coûtait rien aux patrons, ainsi que d'un ensemble d'avantages fort bien décrits par Alfred Marshall dans son célèbre chapitre sur les districts industriels ${ }^{19}$.

13 Comme le produit de l'industrie cotonnière était exporté à $80 \%$ vers 1850 , cette pression constante des marchés extérieurs pour réduire les coûts et augmenter la productivité n'a pu que renforcer les caractéristiques initiales du système productif de ce secteur. C'est ainsi que l'on doit mettre en perspective l'abolition des Corn Laws, dont l'objectif était de préserver ou d'améliorer la compétitivité externe des industries exportatrices britanniques dans la mesure où la rigidité du système monétaire international empêchait tout réajustement de change. Comme le change ne reflétait pas les parités de pouvoir d'achat, mais faisait sans doute, selon l'habitude, paraitre plus chers les prix dans les pays les plus développés, on peut faire l'hypothèse que les produits anglais devaient avoir un handicap de change par rapport aux autres pays ${ }^{20}$. En effet le rapport des prix des exportations britanniques par rapport aux prix français n'évolua favorablement pour la Grande-Bretagne qu'entre 1845 et 1860 , en rapport probablement avec la politique douanière ${ }^{21}$. Faute d'imaginer une inconcevable dévaluation, la seule manière dont les industriels pouvaient peser sur les coûts salariaux était de faire baisser le niveau des dépenses alimentaires. Le groupe de pression industriel qui y poussait était celui de Manchester.

Il s'agissait donc d'améliorer la compétitivité moins par rapport aux autres pays européens que par rapport à l'Inde. La production cotonnière britannique était spécialisée sur les qualités extrêmes. Outre la cotonnade très bon marché à destination de l'Asie, elle vendait en Europe des produits très difficiles à fabriquer pour lesquels elle avait une supériorité technologique, filés de grands numéros, tissus très fins: la concurrence n'était pas en termes de prix ; en fait il n'y avait guère de concurrence. En revanche la concurrence en termes de prix était forte dans le cas des tissus les plus grossiers vendus en Asie où un effet de seuil existait, celui du prix permettant aux tisserands locaux de rester encore sur le marché ou les obligeant à abandonner la production. La spécialisation interne a donc aidé à définir une spécialisation externe qui 
l'a renforcé et qui a été le mode d'insertion de la Grande-Bretagne dans le commerce international. Et l'orientation vers des débouchés commerciaux extra européens non seulement en Asie mais aussi en Amérique latine, combinée à une position excédentaire du marché des capitaux longs, a contribué à développer le rôle de Londres comme marché mondial des capitaux à long terme --comme centre de crédit. Et comme les pays peu développés vers lesquels ces capitaux se dirigeaient étaient des pays où les classes supérieures locales avaient moins confiance dans la solidité de leur économie nationale que dans celle de l'économie du pays dominant, le rôle de Londres comme centre de placement à long terme ne pouvait que s'affirmer. Le rôle de centre de paiement à court terme ne pouvait également que se développer, d'autant que la structure des compensations mondiales favorisait son développement.

\section{Exportations industrielles françaises, structures de production, structures sociales}

15 La Grande-Bretagne a été, en tant qu'économie dominante, le seul pays qui a pu développer ses possibilités de vente, certes dans l'environnement douanier imposé par les autres pays en industrialisation, mais indépendamment de leurs avantages comparatifs. Les industriels des autres pays se sont accommodés des choix initiaux britanniques sur lesquels ils n'avaient pas prise. Les fabricants français se spécialisèrent à l'exportation sur les produits haut de gamme, le seul créneau possible en fonction des marchés disponibles et de la structure de leur industrie. Comme les pays en industrialisation étaient les seuls où existait un pouvoir d'achat croissant, ils représentaient les seuls marchés prometteurs, en dehors des débouchés forcément limités à l'étroitesse des classes supérieures dans les pays moins développés. Mais comme les pays en début d'industrialisation commençaient à mécaniser leurs industries cotonnières, ils prohibaient l'importation des cotonnades de qualité grossière et moyenne. Donc, seules les qualités haut de gamme trouvaient dans les pays en industrialisation un large marché à fort potentiel de croissance. Cela était vrai pour l'ensemble des produits textiles et même pour l'ensemble des produits manufacturés : soieries, cotonnades imprimées alsaciennes, lainages de qualité, tissus dits de "nouveauté" ou articles de Paris. La grande inégalité du partage des gains de pouvoir d'achat dans tous les pays industriels profitait surtout aux couches supérieures des classes moyennes et aux classes supérieures qui étaient la clientèle de ce type de produits. Or ce type de produits correspondait particulièrement bien aux caractéristiques de la demande et de la production sur le marché textile français lui-même, alors qu'il n'était pas fabriqué aux États-Unis, et qu'il était le contraire de la spécialisation britannique.

La structure et l'évolution de la demande du marché intérieur de produits textiles français, entre 1840 et 1914, peuvent être appréhendées en croisant une répartition du revenu national par groupes sociaux et une estimation des grandes masses de dépenses pour chacun de ces groupes à partir des nombreuses enquêtes sur les budgets familiaux ${ }^{22}$. L'hypothèse qui est à la base de cette démarche est que la consommation n'est pas le résultat des comportements d'individus identiques au revenu près, des individus qui, si on les classait par revenu formeraient une pyramide continue et régulière. Au XIX ${ }^{\mathrm{e}}$ siècle, même pour la société la plus homogène, la moins affectée par les discontinuités, qu'était la société américaine, cette dernière image aurait été anachronique. Les sociétés du XIX ${ }^{e}$ siècle étaient segmentées en groupes sociaux dont les comportements de consommation n'étaient pas les mêmes, même pour des individus aux revenus comparables. Car la 
consommation jouait un rôle de marqueur social. Les mécanismes d'imitation étaient importants et soutenaient des réflexes d'envie et des stratégies d'ascension sociale : ils furent un des stimulants à la croissance durant tout le XVIII ${ }^{e}$ et le XIX ${ }^{e}$ siècles. Mais ces mécanismes homogénéisateurs connaissaient leurs limites puisqu'ils mettaient leurs auteurs en danger d'être exclus de leur groupe d'origine sans être acceptés dans un autre groupe. Cela explique le durable maintien d'une forte segmentation des structures de consommation au XIXe siècle.

Une tentative de reconstitution des demandes étrangère et intérieure de produits textiles

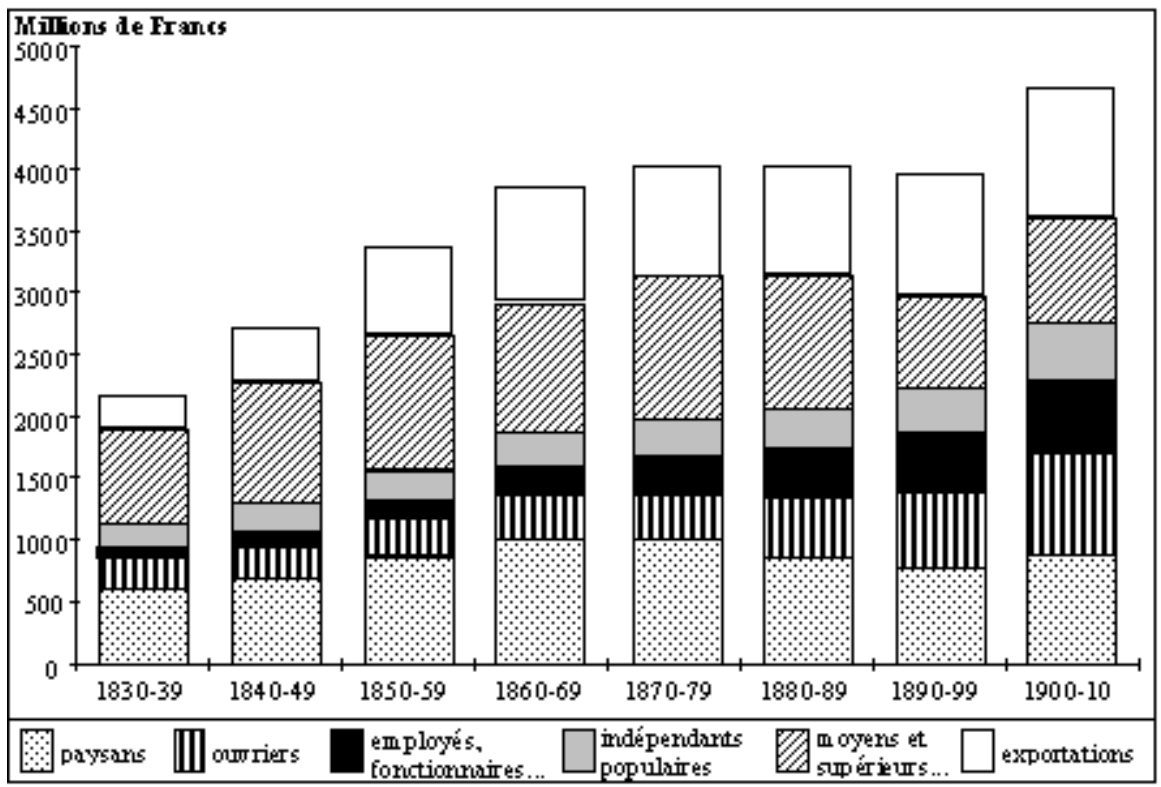

17 La croissance de l'industrie française s'est appuyée de 1820 jusqu'au ralentissement conjoncturel des années 1870 sur la croissance des exportations, plus rapide que celle des ventes à l'intérieur. La demande intérieure était éclatée entre une demande de produits bon marché de la part de populations rurales nombreuses et à pouvoir d'achat en nette augmentation et une demande de produits de qualité de la part des classes supérieures et d'une frange supérieure des classes moyennes, alors qu'au contraire la demande des classes laborieuses urbaines restait peu dynamique. Cette structure et cette dynamique des revenus expliquent l'importance de la production de qualité en France. Elles ont prédisposé ce pays à en être un fournisseur privilégié pour des pays industriels comme les États-Unis et la Grande-Bretagne, où ces produits remplissaient la même fonction de marqueur social et soutenaient une demande en rapide croissance. En effet, la demande de ces produits avait sans doute une plus forte élasticité par rapport aux revenus des acheteurs que par rapport aux prix: leur consommation ne pouvait que rapidement croître dans des pays en voie d'enrichissement. Et la concurrence se faisant, au début du siècle du moins, moins par les prix que par la qualité, il y avait peu de pression à économiser des coûts en travail, payés par ailleurs bon marché en France. Le fréquent émiettement des structures productives, comme dans l'article de Paris, ou la faible mécanisation dans des industries plus concentrées comme la soierie lyonnaise n'étaient donc pas un handicap à la spécialisation internationale sur ce type de créneau et l'incitation à les faire évoluer était faible. En fait la situation allait évoluer au cours du $\mathrm{XIX}^{\mathrm{e}}$ siècle du fait de la volonté des industriels de vendre davantage en élargissant peu à peu l'assise sociale de leur clientèle, c'est-à-dire en vendant à une clientèle prenant de 
plus en plus ses décisions d'achat en fonction du prix. Leur grande innovation a été celle du demi-luxe pour les classes moyennes, des produits dont l'apparence ressemblait aux produits de luxe et qui conféraient à leur consommateur les signes extérieurs de "bourgeoisie", mais dont le prix était beaucoup moins élevé que les vrais produits de luxe. Cette stratégie de produits qui visait à accroître l'élasticité de la demande par rapport au prix en étendant les ventes avec des produits moins chers qui faisaient dire aux esprits chagrins que la qualité baissait toujours ${ }^{23}$, ne pouvait que saper la spécificité des produits qui les distinguait de la production industrielle de grande série. À la fin du XIX siècle, l'évolution devait aboutir à une convergence de la production de biens de consommation vers des qualités moyennes, avec un resserrement de l'éventail des prix, mise à part une production de luxe désormais en cours de marginalisation, mesurée à l'échelle de l'ensemble de la production industrielle.

\section{Le système mondial des compensations et le rôle de Paris comme second centre de paiement au milieu du $\mathrm{XIX} \mathrm{X}^{\mathrm{e}}$ siècle}

Les différents flux d'échanges de marchandises ne se soldaient pas bilatéralement, mais donnaient, dès l'époque moderne, lieu à des compensations dans un système multilatéral de paiements, qui attribuait à certaines places commerciales et bancaires un rôle de centre de paiement. Pour jouer ce rôle au XIX ${ }^{\mathrm{e}}$ siècle, une place devait posséder une infrastructure bancaire capable d'effectuer des opérations à l'échelle internationale, avec des maisons dont la signature était connue dans la communauté commerciale internationale, et avoir une monnaie nationale dont la convertibilité en métal précieux et la stabilité de la définition métal inspiraient une totale confiance. Mais il fallait aussi et surtout que les ramifications du commerce national soient développées et que la structure des soldes commerciaux favorise le développement d'activités de compensation. Cette dernière cause est en fin de compte la principale, car c'est elle qui explique le développement des infrastructures négociantes et bancaires orientées vers les opérations internationales. Ces dernières mettaient longtemps à s'établir et exerçaient ensuite un effet d'attraction qui freinait le développement de places concurrentes. C'est pourquoi entre les trois principaux partenaires du commerce international GrandeBretagne, France et États-Unis ${ }^{24}$, la place de New York ne joua jusqu'en 1913 qu'un rôle très limité de centre de paiement en comparaison de Londres ou de Paris.

Le schéma des compensations peut être tracé en calculant les soldes de balances bilatérales des biens entre les trois principales puissances commerciales et les autres pays regroupés ici, pour des raisons de lisibilité, selon le type de leurs échanges et selon le caractère excédentaire ou déficitaire de leurs balance ${ }^{25}$. En toute rigueur, il conviendrait de calculer les soldes de la balance des transactions courantes, incluant les postes importants qu'étaient déjà les services (marine, tourisme), les transferts d'argent par les migrants définitifs ou saisonniers et la rémunération des capitaux prêtés à l'étranger et y ajouter les soldes de la balance des métaux précieux, représentant les paiements hors compensations. Mais cela reporterait l'élaboration d'un cadre d'ensemble du fonctionnement de l'économie internationale au moment où l'on aura pu mener les recherches minutieuses que cela impliquerait, alors qu'en fait ces flux accentuaient les principaux soldes de marchandises plutôt qu'ils ne les inversaient. On ne tiendra compte 
que du solde du tourisme britannique et américain en France ${ }^{26}$. Les déficits importants de la France envers le reste de l'Europe n'étaient en effet sûrement pas compensés par les dépenses des touristes allemands, russes, scandinaves ou italiens, beaucoup moins nombreux ${ }^{27}$.

En revanche pour la Grande-Bretagne, le solde négatif des flux de marchandises avec les États-Unis était partiellement compensé par les "invisibles" et le revenu des capitaux. Mais la balance des paiements restait déficitaire et s'il y avait néanmoins de massives exportations de capitaux, ce n'était pas contradictoire, car l'équilibre comptable de la balance des paiements n'existe qu'au niveau le plus agrégé : cela supposait simplement une forte compensation des paiements avec les autres balances bilatérales britanniques ${ }^{28}$. La place de Londres était un centre de compensation pour le commerce américain qui avait des créances sur les banquiers de cette ville. Les maisons de négoce angloaméricaines, représentées des deux côtés de l'Atlantique, les y laissaient en dépôt. Ces créances permettaient aux maisons de la place de compenser les déficits commerciaux des États-Unis avec les pays de la Baltique, le Brésil et surtout la Chine. Londres jouait ainsi, outre son rôle dans le crédit et le placement à long terme, un rôle de centre de paiement et de crédit à court terme, car une grande partie des flux d'exportations et d'importations dans le monde était financée par l'intermédiaire de ces opérations de paiement.

Créances liées aux soldes des balances commerciales (1830-1850)

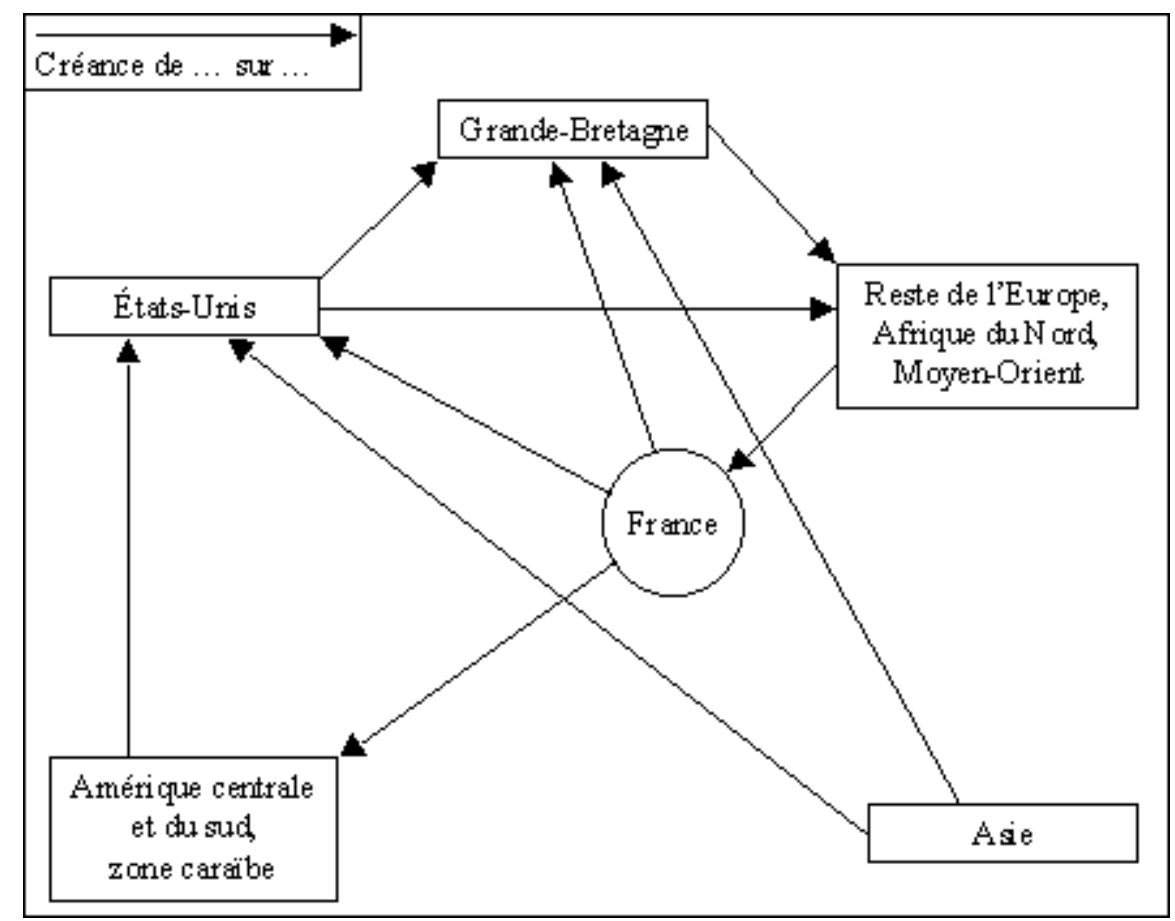

21 Les calculs des soldes de balances des flux de marchandises et de métaux précieux ${ }^{29}$ révèlent qu'au niveau du continent européen, c'était plutôt la place de Paris qui jouait un rôle majeur ${ }^{30}$. En effet, les ventes de vins, d'alcool, de soieries et d'autres produits manufacturés aux États-Unis et en Grande-Bretagne --les deux premiers marchés d'exportation--, assuraient des créances aux négociants français sur ces deux pays, qu'ils laissaient en dépôt chez les banquiers parisiens. Or les États-Unis et la Grande-Bretagne avaient des créances sur l'Europe continentale, surtout sur les pays germaniques. Les 
balances commerciales germaniques étaient déséquilibrées avec la Grande-Bretagne du fait des importantes importations de produits tropicaux comme le café qui étaient réexportés par le négoce britannique. Le commerce des pays germaniques et méditerranéens avec les États-Unis était très déséquilibré parce qu'ils achetaient du coton, la matière première de l'industrialisation, sans pouvoir leur vendre ce qu'ils vendaient aux autres pays européens, c'est-à-dire des produits primaires comme le bois, le blé, les cuirs, le bétail sur pied qui existaient beaucoup moins cher outre-Atlantique et dont de toute façon le coût de transport aurait été prohibitif. Les Allemands ou les Italiens auraient pu exporter aux États-Unis des produits similaires aux produits français, car leurs ouvriers qualifiés possédaient les mêmes savoir-faire techniques que les Français, parfois maîtrisés depuis plus longtemps qu'en France. Mais les fabricants de soieries de Krefeld, Elberfeld, de Toscane, de Lombardie ou l'industrie lainière d'Italie du Nord, pourtant l'une des premières d'Europe, ne parvenaient pas à pénétrer suffisamment le marché américain, seules la soierie de Zurich ou la rubanerie de Bâle y parvenaient, mais elles n'inquiétaient pas encore les Lyonnais ou les Stéphanois, plus intéressés d'ailleurs à enlever à Londres son rôle de marché mondial de la soie brute ${ }^{31}$. Si les Allemands et les Italiens ne parvenaient pas à équilibrer leurs échanges, c'était sans doute aussi parce qu'ils n'avaient pas mis en place des réseaux de commercialisation outre-Atlantique, opération de longue haleine. Leurs réseaux étaient plutôt tournés vers l'Est, par Leipzig qui était une interface avec le marché russe et avec tout le Moyen-Orient. Les réseaux d'approvisionnement du coton, dont les organisateurs cherchaient évidemment à développer aussi les flux de marchandises en sens inverse, étaient dans les mains des Britanniques et des Français. Les Allemands étaient donc endettés vis-à-vis de leurs fournisseurs américains et anglais.

Mais les Allemands, les Italiens et les Belges pouvaient s'acquitter de leurs dettes en s'adressant aux banquiers parisiens qui géraient leurs avoirs. En effet, le Zollverein, les pays italiens, la Belgique, les Pays-Bas exportaient plus en France qu'ils n'importaient, car les Français étaient acheteurs de produits primaires, sans pouvoir développer de fortes ventes de vin ou de produits de "demi-luxe" à la fois parce que les niveaux de vie de ces pays étaient inférieurs aux niveaux de vie américains ou britanniques et parce que leurs industries fabriquaient souvent des produits similaires. Le mouvement des métaux précieux accroissait encore le déficit français sur ces pays.

Une estimation des soldes des balances bilatérales des paiements courants de la France (Millions de francs. Moyennes annuelles 1827-1866)

\begin{tabular}{|l|l|l|l|l|l|}
\hline Balances du... & commerce général (A) & métaux précieux (B) & $\mathrm{A}+\mathrm{B}$ & Tourisme $^{32}$ & Total \\
\hline Grande-Bretagne & 160,7 & -112 & 49 & 70 & 119 \\
\hline États-Unis & 56,5 & -17 & 39 & 8 & 47 \\
\hline Amérique latine & 52,1 & -8 & 44 & & 44 \\
\hline Pays italiens & $-2,1$ & 3 & 1 & & 1 \\
\hline \hline Pays du nord & $-22,4$ & 0 & -22 & & -22 \\
\hline Russie & $-35,5$ & 2 & -34 & & -34 \\
\hline
\end{tabular}




\begin{tabular}{|l|l|l|l|l|l|}
\hline Asie & $-51,7$ & 17 & -35 & & -35 \\
\hline \hline Pays germaniques & $-18,1$ & -22 & -40 & & -40 \\
\hline dont Zollverein & $-26,6$ & -23 & -49 & & -49 \\
\hline Belgique/Pays-Bas & $-65,2$ & -11 & -76 & & -76 \\
\hline
\end{tabular}

Les banquiers allemands, belges et sans doute italiens avaient donc des créances sur Paris. Les soldes inverses se compensaient ainsi aisément en économisant le plus possible les transports de métaux précieux, rendus complexes par l'existence de deux étalons dans le monde. L'installation dès les années 1820 de nombreux banquiers rhénans à Paris correspondit au développement de ces opérations de compensation et de paiements. C'est à la lumière de ce rôle de Paris comme centre international de paiements qu'il faut évaluer le projet des frères Pereire de faire, au début du Second Empire, un emporium continental du quartier de l'Europe à Paris pour réunir toutes les marchandises du continent, centralisées, prêtes à être vendues aux États-Unis et en Grande-Bretagne et, subsidiairement, en Amérique latine. Il s'agissait de faire basculer vers Paris la partie des échanges allemands qui passait par Anvers ou Rotterdam et était médiatisée par les banquiers britanniques ou plutôt par les banquiers allemands installés à Londres, dans la mesure où les banquiers britanniques étaient peu disposés à faire confiance ou crédit aux maisons de négoce allemandes et à développer leurs réseaux en Europe moyenne ${ }^{33}$. Les Pereire pensaient possible d'aligner, grâce aux chemins de fer, les flux commerciaux sur les flux de paiements. Ainsi devait être consacrée l'importance des maisons de banque parisiennes.

En fait à envisager les flux avec plus de précision, il semble que d'autres circuits financiers pouvaient fonctionner, mais la réalité des flux de paiements doit être attestée par des recherches à faire dans les archives bancaires et négociantes. Une reconstitution vraiment fiable de tous les flux demanderait une recherche beaucoup plus développée prenant en compte les statistiques douanières de tous des pays européens, en particulier celles des États italiens. En travaillant seulement à partir des sources françaises, britanniques, allemandes et américaines, on obtient quelques pistes : 
D'autres circuits pouvant utiliser comme financement les créances françaises sur la GrandeBretagne

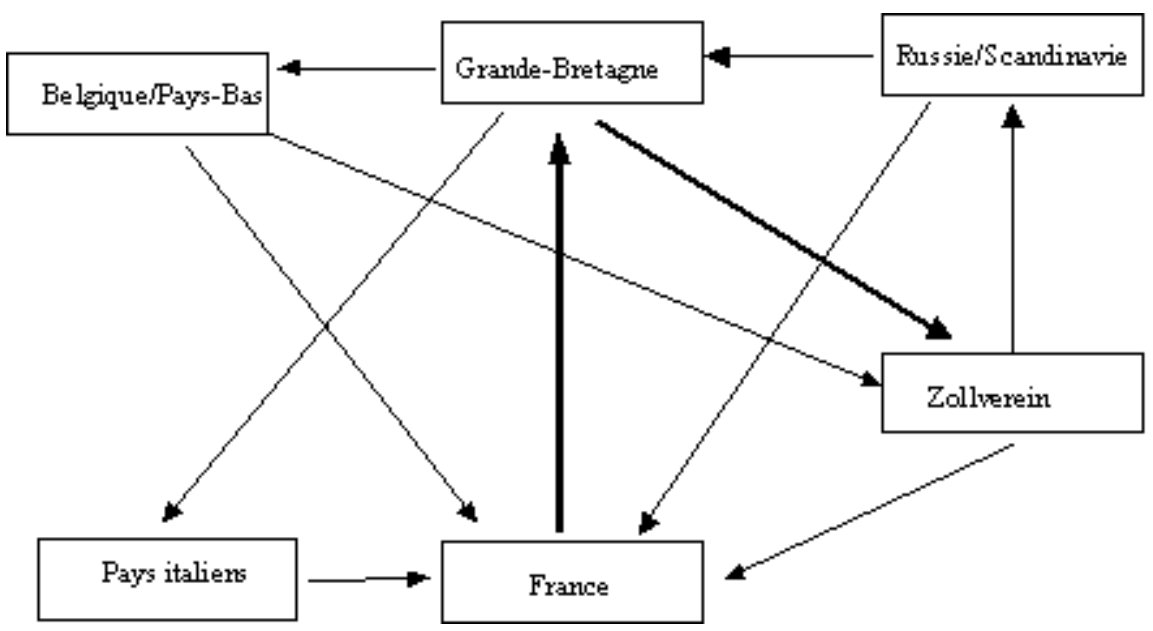

La Belgique, les Pays-Bas et les pays italiens occupaient une position similaire à celle du Zollverein. Ces deux pays avaient des excédents sur la France par leurs ventes de matières premières et de produits agricoles : houille, lin teillé, bestiaux pour le premier, fromages, zinc, laines en masse, étain brut pour les seconds, soies, huile d'olive, bestiaux pour les troisième. Ces différents pays compensaient ainsi leurs déficits sur la Grande-Bretagne. Quant à la Russie et aux pays scandinaves, ils avaient par leurs ventes de matières premières (céréales, fibres textiles, graisses, bois) des excédents considérables, sur la France ${ }^{34}$ et la Grande-Bretagne, qui en dehors de mouvements de capitaux à long terme ne pouvaient être payés que par deux circuits de compensation impliquant les créances sur les commerces britannique et français, selon des circuits multiséculaires. Le circuit le plus simple s'appuyait sur la Grande-Bretagne et le Zollverein. Un circuit secondaire pouvait impliquer les banquiers parisiens (Pays du Nord-France-Grande-BretagneZollverein). Dans les deux cas les banquiers allemands jouaient un rôle majeur, comme Bleichröder ou Mendelssohn de Berlin, ou bien les frères Bethmann de Francfort, qui étaient en relation avec la Russie.

Le rôle de la place de Paris dans les paiements internationaux était renforcé par sa position dans les flux de métaux précieux puisque le bimétallisme du franc permettait de faire communiquer le monde de l'étalon argent --c'est-à-dire les pays germaniques et l'Asie--, avec le monde de l'étalon or centré sur la Grande-Bretagne, et qui, dans les années 1850-1860, ne comprenait guère que le Portugal. Or les paiements en Asie restaient le problème global du système international au milieu du XIX ${ }^{e}$ siècle, puisqu'en dépit du développement de cultures comme le thé en Inde, qui permettait de maintenir à Londres les créances des exportateurs, malgré les guerres de l'Opium, malgré les compensations entre l'Inde et la Chine, le commerce restait encore déséquilibré avec l'Extrême-Orient. Cette position asymétrique du commerce d'Asie était le seul facteur qui empêchait le parfait multilatéralisme du milieu du XIXe siècle. C'est pourquoi les pays asiatiques n'étaient pas un élément "marginal" dans le système des flux internationaux, mais au contraire en eux résidait la possibilité d'un "bouclage" de ce système, qui ne se réalisait pas dans les années 1850-1860, mais qui allait se réaliser en fin de siècle.

Ce système relativement harmonieux tendit à se déstructurer dès les années 1860 parce que deux soldes de balance commerciale qui correspondaient à des flux majeurs se 
modifièrent. Du fait de la guerre de Sécession, le commerce américain connut un fort fléchissement, tant aux exportations --la "famine du coton"--, qu'aux importations. Les exportations françaises vers les États-Unis s'effondrèrent, car les produits de haute qualité sont beaucoup plus sensibles à la conjoncture politique et économique que les produits de qualité ordinaire ${ }^{35}$. Cette chute des ventes de soieries ou de vins était contrebalancée par l'effondrement d'une partie des importations totales de coton, dans la mesure où une autre partie du coton était achetée en Grande-Bretagne. Le solde de la balance française des paiements courants avec les États-Unis ne changea pas de signe, sauf pour la dramatique année 1861 , mais son montant fut fortement réduit ${ }^{36}$, c'est-à-dire que les banquiers parisiens n'avaient plus les mêmes disponibilités en créances sur la place de New York.

Au même moment, les exportateurs français avaient progressé sur les marchés italiens en vendant davantage de produits haut de gamme dans des sociétés dont l'enrichissement avait jusqu'alors tardé à s'affirmer ${ }^{37}$, mais aussi des biens d'équipement en raison de l'accélération de l'industrialisation. Les modalités de la construction ferroviaire française, dominée par l'initiative des groupes financiers, avaient favorisé une reproduction du même modèle dans les pays voisins. Ces mêmes groupes financiers parisiens participèrent dès les années 1850 à la construction des réseaux ferroviaires et ces investissements directs à l'étranger suscitèrent des exportations, ce qui était cohérent avec une évolution de la balance française des paiements courants vers un solde positif ${ }^{38}$. La fonction commerciale et financière de Paris était en tout cas en train de se modifier. Paris était de moins en moins un centre de paiements, un centre de compensation du commerce international. Les banquiers tendaient à s'orienter vers l'investissement direct à l'étranger en Italie, en Espagne, en Autriche.

Désormais donc, dans le dernier tiers du XIX siècle, le système mondial de compensations allait se recentrer sur un seul pôle, Londres. Le rôle de la place de Paris était en train de devenir marginal pour les paiements internationaux ; c'est-à-dire pour les mouvements de capitaux à court terme Cette tendance a évidemment été accentuée par l'inconvertibilité du franc de la guerre de 1870-1871 à 1878, et par la crise des exportations françaises --en particulier celle des produits de luxe et de demi-luxe ${ }^{39}$. Le ralentissement de la croissance des pays industriels pendant la période dite de la "Grande dépression", touchait en effet davantage les revenus de la propriété et de l'entreprise, donc les revenus des classes aisées-, plutôt que les salaires réels qui s'orientaient à la hausse : l'élasticité par rapport au revenu de la consommation de produits de qualité jouait désormais au détriment des exportations françaises. La désagrégation du système d'échanges internationaux du milieu du siècle qui a accompagné les changements de la hiérarchie des niveaux de développement industriel et donc ceux de la géographie de la production mondiale, explique le caractère plus conflictuel des relations économiques internationales durant cette période. Ce dernier eut pour conséquence une intervention accrue des États avec une montée d'un type de protectionnisme très différent de celui de la première moitié du XIX siècle. Celui des années 1820-1850 était "listien" visant à protéger les industries "naissantes" comme les industries cotonnières ou sidérurgiques en train d'aborder leur modernisation. Celui des années 1890-1900 s'efforçait plutôt de protéger les industries déclinantes, avec un effet sans doute plutôt négatif pour la croissance économique ${ }^{40}$ puisqu'il ralentissait la réallocation sectorielle de la main d'œuvre et des capitaux, mais avec un effet social positif puisqu'il permit de limiter le coût social d'une restructuration brutale des appareils productifs. 


\section{L'émergence du système commercial anglo-saxon de la fin du XIX ${ }^{\mathrm{e}}$ siècle}

À partir des années 1890, un nouveau système se reconstruisit au plan commercial, au plan des compensations, au plan monétaire. La France n'a pas retrouvé, en termes relatifs, la position commerciale qu'elle avait occupée, alors qu'après 1890 l'Allemagne devenait le second exportateur mondial de produits manufacturés et se rapprochait peu à peu du niveau britannique. En dehors de quelques travaux majeurs, mais partiels dans leur cadrage chronologique ${ }^{41}$, il manque une étude approfondie qui évaluerait les composantes de l'évolution des échanges français à partir de 1880. Les analyses d'Alfred Maizels ${ }^{42}$ restent une référence : elles suggèrent des hypothèses qui, intuitivement, sont plausibles. La croissance du commerce français en fin de XIX siècle aurait été une réponse passive à la reprise de la croissance mondiale plutôt que le signe d'un dynamisme particulier. La diversification en terme de marchés aurait été faible : c'est-à-dire qu'il n'y a guère eu de report des marchés à faible potentiel de croissance vers les marchés les plus prometteurs. La diversification en terme de produits n'aurait pas été non plus très satisfaisante, car il ne faut pas surestimer les exportations des secteurs de pointe comme l'automobile, par rapport aux vieux secteurs d'exportation à faible croissance de productivité, pour lesquels la demande mondiale commençait à devenir de moins en moins élastique, comme les industries de la soie.

Part dans les exportations mondiales de produits manufacturés ${ }^{43}$

\begin{tabular}{|l|l|l|}
\hline & 1899 & 1913 \\
\hline Royaume-Uni & 33,2 & 30,2 \\
\hline Allemagne & 22,4 & 26,6 \\
\hline États-Unis & 11,7 & 13,0 \\
\hline France & 14,4 & 12,1 \\
\hline Belgique-Luxembourg & 5,5 & 5,0 \\
\hline Italie & 3,6 & 3,3 \\
\hline \hline Suisse & 4,0 & 3,1 \\
\hline
\end{tabular}

31 Dans les années 1890 se mit en place un nouveau circuit de compensation axé sur des positions complémentaires du Royaume-Uni, des pays neufs fournisseurs de produits agricoles, des États-Unis et de l'Inde, maillon important dans le bouclage du système. C'était un système anglo-saxon, que Samuel Berrick Saul a très bien décrit dans un ouvrage qui reste une référence ${ }^{44}$ et dont nous ne reprenons pas l'exposition. Il consacrait le rôle central du Royaume-Uni dans les relations économiques mondiales qui était renforcé par le développement des marchés à terme de matières premières centralisés en Grande-Bretagne. Le recul de la livre sterling comme liquidité 
internationale du fait du développement des pratiques de l'étalon or et des paiements bancaires dans d'autres devises entre les banques mères et leurs filiales étrangères n'entamait pas cette position dominante. Le commerce britannique restait le plus important du monde. Si l'Allemagne allait approcher en valeur des exportations de produits manufacturés le niveau du Royaume-Uni à partir de 1910, en revanche l'économie britannique restait la première importatrice mondiale de produits primaires. Elle restait le partenaire le plus important pour la plupart des pays. Elle était surtout celui dont les soldes des balances des transactions courantes et des métaux précieux étaient les plus déséquilibrés, ce qui la situait toujours au centre du multilatéralisme mondial.

La balance britannique de 1910 était déficitaire. Les pays recensés dans le schéma de Saul faisaient apparaître 145 millions de livres de dettes et 118 millions de créances. La compensation des paiements mondiaux se faisait donc largement entre ces créances et ces dettes. Les principales dettes du Royaume-Uni étaient envers les États-Unis, l'Europe continentale et les pays neufs fournisseurs agricoles (Canada surtout, et à la fin du XIX siècle Australie et Argentine). L'Europe continentale était, vingt ans auparavant, le principal créancier; son surplus diminua après 1900 de par la croissance de ses importations de charbon d'Angleterre. Après 1900, le rôle des États-Unis se modifia également, avec une réduction du déficit britannique, car la Grande-Bretagne achetait de moins en moins son alimentation dans ce pays, se tournant vers l'Argentine et le Canada. En revanche dans ces deux derniers pays, le solde commercial britannique était d'autant plus déficitaire que les exportations américaines de produits manufacturés tendaient à y remplacer les britanniques.

Le schéma de S. B. Saul ${ }^{45}$

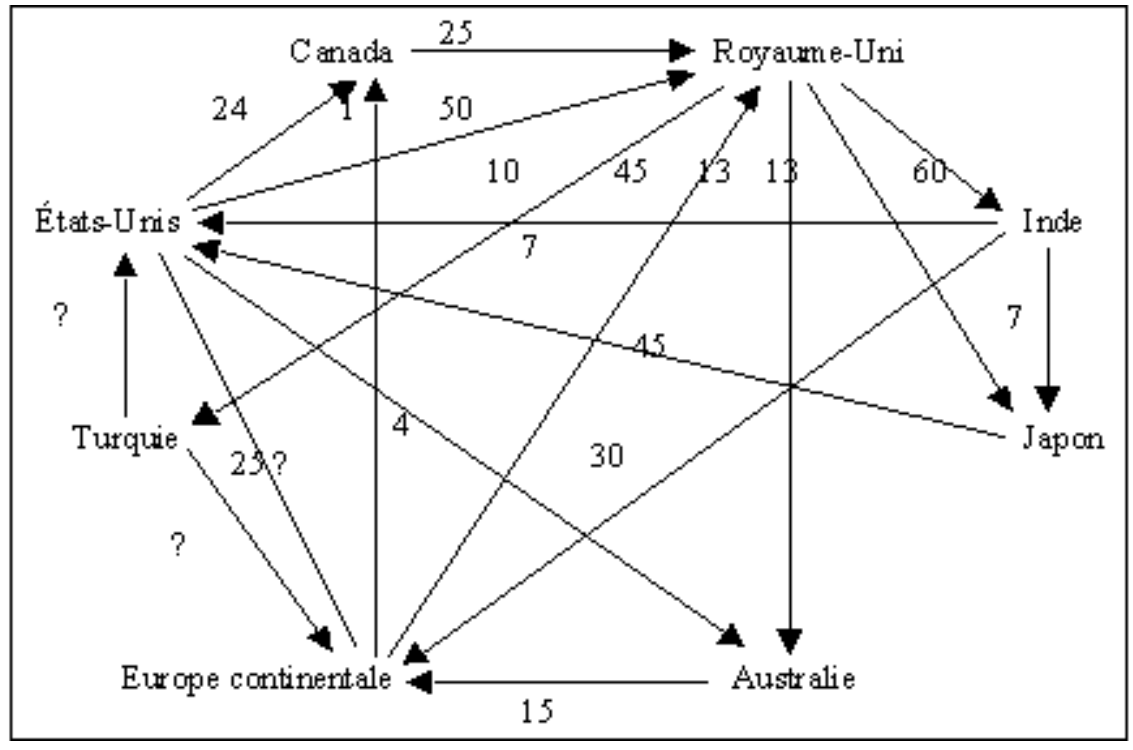

En 1910, le Royaume-Uni compensait en gros 120 millions de dettes, envers les États-Unis, l'Europe continentale et le Canada, grâce à ses surplus sur l'Inde, sur l'Australie, le Japon et la Chine. La dette canadienne était transférée aux États-Unis puisque le Canada avait une balance positive avec la Grande-Bretagne et négative sur les États-Unis. Les ÉtatsUnis étaient toujours le second pivot du système des compensations en tant que créancier important du Royaume-Uni. Or les États-Unis avaient un déficit envers l'Inde et le Japon, ce qui permettait une compensation directe. L'Inde apparaissait donc comme le troisième 
pivot du système : c'était la vente de produits manufacturés britanniques en Inde qui assurait le fonctionnement de l'économie internationale, favorisée par l'implantation des réseaux marchands britanniques dans ce pays et par la faible pénétration des autres pays exportateurs sur ce marché.

Mais une partie plus importante de la dette restait compensée au travers de l'Europe continentale, qui, à en croire le schéma de Saul, pouvait utiliser 30 à 40 millions des créances qu'elle avait sur la Grande-Bretagne pour payer ses dettes envers l'Australie, l'Inde et sans doute la Turquie, toutes trois endettées envers le Royaume-Uni.

Le schéma de S. B. Saul ne décontractait pas l'ensemble "Europe continentale", qui comprenait des pays à structure commerciale très différente, qu'il créditait globalement de surplus, en 1910 sur la Grande-Bretagne et sur les États-Unis. Son texte suggère des nuances importantes comme le rôle de fournisseurs de dollars en Europe qu'était celui de l'Italie, de l'Autriche-Hongrie grâce aux envois de leurs ressortissants émigrés, et, dans une moindre mesure, de la France ${ }^{46}$. Mais les pays qui pouvaient jouer un rôle dans le système global de compensations étaient seulement ceux qui avaient des créances sur le Royaume-Uni. Une recherche approfondie serait nécessaire pour dégager les soldes de balances bilatérales des paiements courants avec tous les pays européens. Les soldes des balances des biens peuvent néanmoins aider à formuler des hypothèses.

Les balances commerciales bilatérales du Royaume-Uni en 1909 (en millions de marks) ${ }^{47}$

\begin{tabular}{|l|l|l|l|}
\hline En 1909 & Importations britanniques de & Exportations britanniques vers & Solde \\
\hline Russie & 36898 & 18326 & -18572 \\
\hline Suède & 9245 & 7114 & -2131 \\
\hline Norvège & 6574 & 3835 & -2739 \\
\hline Danemark & 19427 & 5705 & -13722 \\
\hline Allemagne & 40115 & 47169 & 7054 \\
\hline Pays-Bas & 37372 & 16304 & -21068 \\
\hline Belgique & 29218 & 19285 & -9933 \\
\hline France & 50691 & 31515 & -19176 \\
\hline Portugal & 2913 & 2777 & -136 \\
\hline Espagne & 13363 & 5352 & -8011 \\
\hline Italie & 3634 & 13275 & 9641 \\
\hline Autriche-Hongrie & 1208 & 4333 & 3125 \\
\hline Grèce & 1613 & 1514 & -99 \\
\hline Bulgarie & 151 & 902 & 751 \\
\hline
\end{tabular}




\begin{tabular}{|l|l|l|l|}
\hline Roumanie & 3395 & 1750 & -1645 \\
\hline Total & 255817 & 179156 & -76661 \\
\hline
\end{tabular}

Bien que S. B. Saul insiste sur les progrès commerciaux de l'Allemagne sur les marchés européens et sur l'importance de ses "invisibles" qui semblent pourtant n'avoir fait l'objet d'aucune évaluation, il semble plutôt que ce pays restait en déficit en 1909 et n'était donc pas partie prenante au système de compensation anglo-saxon centré sur la Grande-Bretagne. La hiérarchie des créanciers commerciaux du Royaume-Uni mettait en revanche toujours en valeur le rôle de la Russie et des pays du Nord --des Pays-Bas à la Scandinavie--, le vieux schéma. Un dernier point d'interrogation est la position de la balance française par rapport à la Russie, qui présentait un considérable déficit commercial, réduit au niveau de la balance des paiements courants par les revenus des capitaux. Saul évoque l'idée que ces revenus financiers suffisaient à changer de signe le solde de la balance des paiements courants : cela demanderait une étude spécifique.

Créances commerciales continentales sur le Royaume-Uni en 1909 (tableau résumé en millions de marks)

\begin{tabular}{|l|l||l|}
\hline Russie et Scandinavie (dont Danemark) & 37164 & $38 \%$ \\
\hline Pays-Bas et Belgique & 31001 & $32 \%$ \\
\hline France & 19176 & $20 \%$ \\
\hline \hline Portugal et Espagne & 8147 & $8 \%$ \\
\hline Grèce, Roumanie & 1744 & $2 \%$ \\
\hline \hline Total des créances sur le Royaume-Uni & 97232 & $100 \%$ \\
\hline
\end{tabular}

Il y avait donc bien dans les flux de paiements à court terme une certaine marginalisation de la France, qui n'était plus qu'un centre de compensation secondaire sans avoir pour autant perdu totalement cette fonction, mais qui était devenue un centre de financement long, dans une division du travail avec Londres. Si on force le trait, Paris plaçait des emprunts d'État, Londres finançait plutôt le développement de la production internationale des matières premières et des grands produits agricoles. Sur le plan monétaire, la complémentarité des rôles de la France et de la Grande-Bretagne s'était également affirmée entre la Banque d'Angleterre, régulateur général, et la Banque de France, banque de réserve métal.

La position de l'économie française dans l'économie internationale est passée d'une relative centralité au milieu du XIX siècle à une position secondaire au début du $\mathrm{XX}^{\mathrm{e}}$ siècle en ce qui concerne les flux de marchandises et de paiements. Ce changement a été la résultante d'évolutions internes et des mutations des économies des autres pays, en fait 
de la combinaison des deux, c'est-à-dire de la capacité d'adaptation de l'appareil productif interne à suivre ou mieux encore à anticiper les comportements des marchés et de la concurrence, à créer des niches sur des marchés porteurs à défaut de pouvoir s'imposer dans une concurrence frontale par la compétitivité-prix pour les grands produits. Le protectionnisme de la fin du XIX siècle ne semble pas avoir favorisé une division du travail entre les exportateurs de produits manufacturés dont la structure des exportations restait très similaire au niveau des grandes branches industrielles (textile, sidérurgie, construction mécanique...).

La formalisation des changements commerciaux en croissance de la demande, compétitivité et diversification, classique depuis Maizels ${ }^{48}$, reste un outil pertinent d'analyse qui mériterait qu'on lui applique un effort plus poussé d'estimation statistique. Si l'on comprend certains éléments de la mauvaise diversification géographique, comme l'importance croissante, dans les circuits commerciaux et financiers internationaux, de l'Inde, du Canada, de l'Australie qui défavorisait les intérêts français, d'autres demandent des recherches plus poussées.

La forte régression des exportations françaises sur les marchés latino-américains était sans aucun doute liée à un dépérissement des réseaux commerciaux qui avaient permis au milieu du siècle d'y avoir de bonnes positions. La disjonction géographique entre les flux d'exportations de capitaux et les marchés d'exportations, qui peut sembler au premier abord un facteur négatif, supposait un développement de mouvements multilatéraux de capitaux. Ainsi le fort déficit commercial français envers la Russie, s'il n'était pas plus que compensé au niveau de la balance des paiements courants, était totalement contradictoire avec un mouvement de capitaux supposant des excédents, en dehors de compensations multilatérales avec des pays tiers. Cette apparente contradiction laisse à supposer l'existence d'un circuit de compensation entre un créancier de la Russie qui soit en même temps en position de débiteur envers la France, comme l'Allemagne jusqu'en 1908, année où la progression de ses exportations lui permit de devenir créditrice sur l'économie française.

Quant à l'évaluation de la "compétitivité" d'un pays qu'intuitivement l'on associe à l'évolution de sa productivité comparée à celle des pays concurrents, il convient de se rappeler que cette expression qui n'a plus guère de sens en régime de changes flottants puisqu'elle dépend des taux de change, n'en conserve un pour le XIXe siècle que dans la mesure où l'on postule que l'étalon or assurait une "vérité" et une égalisation des prix et des coûts, qui dans la réalité ne se réalisait sûrement pas. Avant même les années 1920 où l'influence des phénomènes monétaires allait devenir patente, leur effet subtil et sans doute peu apparent aux yeux des contemporains ${ }^{49}$ mériterait aussi d'être pris en compte pour faire un "audit" du commerce des principaux pays en 1913.

\section{ANNEXES}


Note 10 : Calculs en valeur d'après la compilation et l'harmonisation des sources officielles par Bernard HARMS, "Volkswirtschaft und Weltwirtschaft. Versuch einer Begründung einer Weltwirtschaftslehre", dans Probleme der Weltwirtschaft. Schriften des Instituts für Seeverkehr und Weltwirtschaft an der Universität Kiel, VI, Jena, G. Fischer, 1912, p. 183.

\begin{tabular}{|l|l|l|l|l|l|l|}
\hline & & Importations & & & Exportations & \\
\hline & $\begin{array}{l}\text { Produits } \\
\text { agricoles }\end{array}$ & $\begin{array}{l}\text { Matières } \\
\text { premières } \\
\text { industrielles }\end{array}$ & $\begin{array}{l}\text { Produits } \\
\text { manufacturés }\end{array}$ & $\begin{array}{l}\text { Produits } \\
\text { agricoles }\end{array}$ & $\begin{array}{l}\text { Matières } \\
\text { premières } \\
\text { industrielles }\end{array}$ & $\begin{array}{l}\text { Produits } \\
\text { manufacturés. }\end{array}$ \\
\hline 1872 & $27 \%$ & $51 \%$ & $22 \%$ & $22 \%$ & $34 \%$ & $44 \%$ \\
\hline 1890 & $34 \%$ & $43 \%$ & $24 \%$ & $14 \%$ & $21 \%$ & $65 \%$ \\
\hline 1900 & $31 \%$ & $49 \%$ & $21 \%$ & $11 \%$ & $24 \%$ & $65 \%$ \\
\hline 1910 & $28 \%$ & $57 \%$ & $15 \%$ & $10 \%$ & $26 \%$ & $64 \%$ \\
\hline
\end{tabular}

* y compris les demi-produits.

Note 36: Balances France-États-Unis (en millions de francs).

\begin{tabular}{|l|l|l|l|l|}
\hline & Marchandises & Métaux & Tourisme & Total \\
\hline 1857 & 177,1 & $-9,6$ & 12,3 & 179,8 \\
\hline 1858 & 108,7 & $-27,4$ & 12,9 & 94,2 \\
\hline 1859 & 218,2 & $-86,0$ & 20,2 & 152,4 \\
\hline 1860 & 102,1 & $-76,5$ & 15,4 & 41,0 \\
\hline 1861 & $-280,4$ & 4,1 & 13,8 & $-262,5$ \\
\hline 1862 & 28,8 & $-1,2$ & 12,7 & 40,3 \\
\hline 1863 & 15,7 & 1,4 & 13,6 & 30,7 \\
\hline 1864 & 25,7 & $-1,2$ & 15,2 & 39,7 \\
\hline 1865 & 77,2 & $-7,7$ & 19,8 & 89,3 \\
\hline 1866 & 26,3 & $-20,4$ & 22,2 & 28,1 \\
\hline
\end{tabular}

Note 38 : Balances France-Royaume d'Italie (en millions de francs).

Marchandises Métaux Total. 


\begin{tabular}{|l|l|l|l|}
\hline 1857 & $-3,3$ & 1,8 & $-1,5$ \\
\hline 1858 & 14,4 & $-20,5$ & $-6,1$ \\
\hline 1859 & 80,6 & $-2,5$ & 78,1 \\
\hline 1860 & 49,1 & $-3,1$ & 46,0 \\
\hline 1861 & 80,5 & 47,1 & 127,6 \\
\hline 1862 & 48,2 & 58,3 & 106,5 \\
\hline 1863 & 107,0 & 102,1 & 209,1 \\
\hline 1864 & 131,6 & $-1,7$ & 129,9 \\
\hline 1865 & 131,5 & 16,0 & 147,5 \\
\hline 1866 & 73,5 & $-91,8$ \\
\hline
\end{tabular}

Note 48 : On se rappellera les estimations de Maizels pour évaluer les évolutions entre 1899 et 1913. Ils peuvent permettre de décomposer la croissance des exportations de produits manufacturés selon les contributions suivantes:

\begin{tabular}{|l|l|l|l|l|l|}
\hline En valeurs relatives & G.B. & France & Allemagne & États-Unis & Monde. \\
\hline croissance du marché & $77 \%$ & $78 \%$ & $77 \%$ & $76 \%$ & $76 \%$ \\
\hline diversification & $-2 \%$ & $-14 \%$ & $3 \%$ & $40 \%$ & $0 \%$ \\
\hline compétitivité & $-27 \%$ & $-6 \%$ & $42 \%$ & $-17 \%$ & $0 \%$ \\
\hline Croissance des exportations & $48 \%$ & $58 \%$ & $122 \%$ & $100 \%$ & $76 \%$ \\
\hline
\end{tabular}

calculs d'après Alfred MAIZELS, Industrial Growth..., ouv. cité, pp. 199-201.

\section{NOTES}

1. Samuel Berrick SAUL, Studies in British Overseas Trade, Liverpool, Liverpool University Press, 1960.

2. Patrick VERLEY, L'échelle du monde, Paris, Éditions Gallimard, 1997, pp466-469.

3. Carlo PONI, "Mode et innovation: les stratégies des marchands de soie de Lyon au XVIII siècle", dans Revue d'histoire moderne et contemporaine, tome 45, n 3, juillet-septembre 1998.

4. Samuel Berrick SAUL, Studies in British Overseas Trade, ouvcité, p7, propose un schéma centré sur la Grande-Bretagne pour le milieu du XVIII siècle. 
5. La thèse de doctorat en cours de Nadège Sougy, sous la direction d'Anne-Lise Head et de Denis Woronoff, s'attache à étudier ces politiques de valorisation de leur production par les entreprises houillères, avec une démarche de création de nomenclatures de produits selon leur qualité.

6. Les Tableaux du commerce de la France comprenaient déjà dans les années 1850-1860 un nombre considérable d'items --de l'ordre de 800--, tant aux importations qu'aux exportations et ils ne rendaient que médiocrement compte des différences de qualité entre produits similaires.

7. Patrick VERLEY, L'échelle du monde, ouvcité, pp605 et suivantes.

8. C'est-à-dire très fins, puisque le numéro anglais indique le nombre d'écheveaux de 840 yards soit 768,10 mètres, répartis en 7 échevettes de 109,72 mètres, par livre-poids de 453 grammes.

9. La preuve de ce caractère mal informé des marchés était l'existence jusqu'au milieu du XIX ${ }^{\mathrm{e}}$ siècle de crises commerciales régulières qui étaient comme leur nom l'indiquait des crises nées dans le processus de commercialisation et non dans celui de production. Voir Patrick VERLEY, “Crises économiques", dans Encyclopedia Universalis, réédition, 1997.

10. Voir tableau en annexe.

11. D'après Bernard HARMS, "Volkswirtschaft und Weltwirtschaft...", artcité, p207, les produits fabriqués ne constituaient que $24 \%$ en 1900 , que $29 \%$ de la valeur des exportations spéciales américaines en 1910.

12. Cet "apparent" paradoxe fait évidemment penser au paradoxe de Leontief qui explique pourquoi dans les années 1950 les États-Unis avaient un avantage comparatif pour des biens à forte intensité en travail et à faible intensité en capital.

13. Maurice LÉVY-LEBOYER, Les banques européennes et l'industrialisation internationale dans la première moitié du XIXe siècle, Paris, Presses universitaires de France, 1964.

14. Patrick VERLEY, L'échelle du monde, ouvcité.

15. Eli HECKSCHER, "The Effects of Foreign Trade on the Distribution of Income", 1919, repris dans Howard Sylvester ELLIS et Lloyd A. METZLER (eds), Readings in the Theory of International Trade, Philadelphie, Blakiston, 1949 ; Bertil OHLIN, International and Interregional Trade, Cambridge Mass., Harvard University Press, 1933.

16. Nick FR. CRAFTS, British Economic Growth during the Industrial Revolution, Oxford, Clarendon Press, 1985 ; Peter H. LINDERT et Jeffrey G. WILLIAMSON, "Reinterpreting Britain's Social Tables, 1688-1913", dans Explorations in Economic History, 1983, pp94-109; Peter H. LINDERT et Jeffrey G. WILLIAMSON, "English Workers' Living Standards During the Industrial Revolution: A New Look", dans Economic HistoryReview, 2thS., Volume 36, n 1, 1983 (b), pp1-25 ; Patrick VERLEY, L'échelle du monde, ouvcité, pp327 et suivantes.

17. Neil McKENDRICK, John BREWER, John Harold PLUMB, The Birth of a Consumer Society. The Commercialization of Eighteenth Century England, Londres, Europa Publications, 1982 ; John BREWER et Roy PORTER (ed.), Consumption and the World of Goods, Londres/New York, Routledge, 1993.

18. Patrick VERLEY, L'échelle du monde, ouvcité, p561, tableau 89.

19. Alfred MARSHALL, Principles of economics, Londres, MacMillan \& Co, 1890, traduction française Principes d'économie politique, Paris, VGiard et EBrière, 1906-1909, 2 tomes en 3 volumes, reprint Paris/Londres/New York, Gordon \& Breach, 1971, 2 volumes.

20. Les études sur ce sujet font défaut.

21. Patrick VERLEY, L'échelle du monde, ouvcité, p583, graphique 64.

22. Cette recherche a été exposée dans Patrick Verley, L'échelle du monde, ouvcité, pp342 et suivantes.

23. Patrick VERLEY, "Essor et déclin des industries du luxe et du demi-luxe au XIX siècle", dans Jacques MARSEILLE [dir.], Le luxe en France du siècle des Lumières à nos jours, actes du colloque de Paris, 9 et 10 décembre 1998, Paris, Association pour le développement de l'histoire économique, 1999.

24. Jusqu'à 1870, il est difficile de considérer le commerce "allemand" comme un ensemble homogène, bien que dans le Zollverein il y eût déjà une certaine unification de l'espace économique. Il subsistait ainsi deux zones monétaires et les villes hanséatiques restaient en 
dehors du Zollverein. En outre il n'y avait pas de centralisation des paiements sur un seul centre, puisque les opérations étaient partagées entre Hambourg, Cologne, Francfort et Berlin. Dans les années 1870, le commerce allemand allait en revanche dépasser en valeur le commerce français. Néanmoins Bodo von BORRIES, Deutschlands Aussenhandel 1836 bis 1856. Eine statistische Untersuchung zur Frühindustrialisierung, Stuttgart, GFischer Verlag, 1970 ; et Dagmar SOLEYMANI, Les échanges commerciaux entre la France et les États allemands 1834-1869, thèse de doctorat d'histoire sous la direction de François Crouzet, Université Paris 4, 1991, envisagent cet ensemble qu'est le commerce "allemand" avant 1871.

25. On peut hésiter entre les soldes du commerce spécial et ceux du commerce général. Le transit peut recouvrir des situations diverses : un paiement direct à un exportateur étranger, ou un paiement à un exportateur national qui effectue ensuite une opération de compensation, ou un simple paiement à un négociant national s'il s'agit de produits coloniaux issus de colonies n'ayant pas de système économique propre. Ainsi l'exportation de 8 millions de francs de coton en laine de la France vers le Zollverein en 1858 recouvre sans aucun doute des achats à des négociants français pour du coton importé des États-Unis par Le Havre, l'opération étant financée par des banquiers parisiens comme Hottinguer qui devaient percevoir les paiements des Allemands et effectuer grâce à leurs correspondants vers les États-Unis les paiements aux fournisseurs. En revanche les exportations de sucre brut antillais qui dans les années 1860 allaient pour un tiers vers les villes hanséatiques étaient physiquement un "transit", mais financièrement représentaient en fait un paiement final au commerce français. Dans les deux cas, ces opérations de transit doivent être prises en compte pour comprendre comment s'effectuaient les opérations de paiement. On utilisera les soldes du commerce général.

26. Maurice LÉVY-LEBOYER, "La balance des paiements et l'exportation des capitaux français", dans Maurice LÉVY-LEBOYER, La position internationale de la France. Aspects économiques et financiers $X I X^{e}-X X^{e}$ siècles, Paris, Éditions de l'École des hautes études en sciences sociales, 1977. Les transferts liés au tourisme, non négligeables dès les années 1830, se gonflèrent à partir des années 1850 , les touristes anglais étaient bien sûr majoritaires ; le tourisme américain restait très limité.

27. Pour les Italiens et les Belges, le rapatriement des revenus du travail de la part des travailleurs saisonniers allait au contraire dans le sens du solde commercial.

28. C'est ce que laissent supposer les postes de la balance des paiements anglo-américaine reconstruits par Samuel Berrick SAUL, Studies in British Overseas Trade, ouvcité, p16.

29. Patrick VERLEY, L'échelle du monde, ouvcité, pp605 et suivantes. Nous nous fions, pour les échanges français, aux données des Tableaux décennaux du commerce de la France avec ses colonies et les puissances étrangères, qui, comme c'est souvent le cas, peuvent donner des résultats qui divergent des statistiques étrangères. C'est ainsi que nous considérons que la France avait un solde positif dans les années 1850 sur les pays allemands, alors que von Borries conteste les chiffres de l'administration française pour 1851 et considère que le solde était déjà négatif ; Bodo von BORRIES, Deutschlands Aussenhandel..., ouvcité, pp186-189. Dagmar Soleymani n'obtient pas non plus les mêmes soldes.

30. Maurice LÉVY-LEBOYER, Les banques européennes et l'industrialisation internationale dans la première moitié $\mathrm{du} X I X^{e}$ siècle, ouvcité.

31. Le rapport de force est révélateur : dans les années 1860, sur une consommation mondiale de soie de l'ordre de 44 millions de livres-poids, la France en manufacturait 15 millions de livres, le Royaume-Uni 4,9, les pays allemands 2,1 et la Suisse 1,6 millions. Pour les problèmes posés par le fait de la centralisation du marché des soies à Londres, voir la "Note écrite de la Chambre de commerce de Lyon, adoptée le 20 juin 1860", dans Enquête sur le Traité de commerce avec l'Angleterre , Enquête... Industries textiles, Chanvre, jute et lin. Soies et soieries..., Paris, Imprimerie nationale, 1861, pp558-559, et la "séance du lundi 13 août 1860", idem, pp575-578. 
32. Nous avons utilisé les estimations des revenus du tourisme de Maurice LÉVY-LEBOYER, "La balance des paiements...", artcité. Nous avons considéré que les Britanniques avaient été responsables des trois quarts des dépenses des touristes non-américains et avons soustrait de cette somme $15 \%$ pour tenir compte des dépenses des touristes français en Grande-Bretagne.

33. Stanley David CHAPMAN, Merchant Enterprise in Britain from the Industrial Revolution to World War I, Cambridge, Cambridge University Press, 1992.

34. On notera que jusqu'à la réforme de 1857 , les statistiques françaises ne relevaient pas les origines premières ou les destinations finales des marchandises, mais les origines et destinations immédiates : ainsi les échanges avec les villes hanséatiques recouvraient des flux qui pouvaient également concerner la Russie ou l'Autriche. Cette pratique enlève de la précision à l'appréciation du commerce continental.

35. En outre le système des acheteurs des grossistes de New York, qui venaient une fois l'an à Paris et à Lyon faire leurs achats et revendaient aux enchères pour tout le pays ensuite à New York, fut cassé par la guerre. Il ne semble pas qu'il ait été repris ensuite ; voir pour la description de cette pratique Jeanne GAILLARD, Paris, la ville, 1852-1870, Paris, Librairie Honoré Champion, 1977.

36. Voir tableau en annexe.

37. Dagmar Soleymani explique une évolution semblable en Allemagne avec l'essor des exportations de textile à partir de 1850 par la hausse des revenus et par la réduction des coûts de transport grâce au chemin de fer; Dagmar SOLEYMANI, Les échanges commerciaux..., ouvcité, tome 1, p251.

38. . Voir tableau en annexe.

39. Patrick VERLEY, "Dynamique des marchés et croissance industrielle", dans Maurice LÉVYLEBOYER [dir.], Histoire de la France industrielle, Paris, Éditions Larousse, 1996.

40. Le mémoire d'habilitation de Jean-Pierre Dormois, non encore publié, consacré à la relation entre les productivités industrielles et le protectionnisme aboutit à cette conclusion.

41. Albert BRODER, "Les échanges extérieurs et la longue stagnation" et "L'or et la balance des paiements pendant la longue stagnation", dans Yves BRETON, Albert BRODER et Michel LUTFALLA, La longue stagnation en France. L'autre grande dépression 1873-1897, Paris, Éditions Économica, 1997.

42. Alfred MAIZELS, Industrial Growth and World Trade, Cambridge, Cambridge University Press, 1971, pp476 à 484 .

43. Source : idem, p189.

44. Samuel Berrick SAUL, Studies in British Overseas Trade, ouvcité, chapitres 2 et 3.

45. Idem, pp56-58.

46. Idem, pp51-52.

47. calculs d'après les données de Bernard HARMS, "Volkswirtschaft und Weltwirtschaft...", artcité, p172.

48. Voir tableau en annexe.

49. Ils étaient surtout sensibles à la dévalorisation du métal argent qui dopait les exportations des pays à étalon argent par la baisse de leurs prix, mais enchérissait leurs importations et leurs dettes extérieures mesurées en sterling ou en francs... 


\section{RÉSUMÉS}

L'objet de la communication est de tenter de dégager la structure -c'est-à-dire la morphologie de la logique de l'organisation des flux, dans le système commercial international au XIX ${ }^{\mathrm{e}}$ siècle. Il est fait l'hypothèse que le caractère peu conflictuel des relations internationales politiques au $\mathrm{XIX}^{\mathrm{e}}$ siècle, qui tranche avec le siècle précédent, est favorisé par des relations commerciales et financières fondées sur une division du travail élémentaire et non contestée entre les partenaires. Ces spécialisations dans la division internationale du travail sont fondées sur des avantages comparatifs et sur des spécificités antérieures, qu'elles confortent ensuite. On fait ensuite l'hypothèse que la configuration des structures des soldes de balances des paiements courants, qui sont le résultat de la combinaison des différents flux du haut de la balance, explique les mouvements de capitaux à court terme qui servent à effectuer des compensations, et, par voie de conséquence la polarisation des activités de paiements dans quelques centres. L'interdépendance de toutes les lignes dans le document comptable qu'est la balance des paiements suggère l'interdépendance des flux dans la réalité. Cette hypothèse, à vérifier dans les faits, aboutit à décrire un système international simple et cohérent au milieu du XIX ${ }^{e}$ siècle, assurant une position centrale au commerce et à la banque françaises.

The purpose of this paper is to try and make out the structure --i.e. the inner logic of the organisation of flows-- in the international trade system in the $19^{\text {th }}$-century. It is assumed that the relatively non-conflicting aspect of political international relations in the $19^{\text {th }}$-century, contrasting with the previous century, was favoured by commercial and financial relations relying on an elementary division of labour which was unchallenged by the partners. This specialisation in the international division of labour relied on comparative advantages and on older specialities, which were thus reinforced. The hypothesis is then made that the structures of the balances of current payments, which were the result of the combination of the different longterm flows, account for the short-term capital flows whose purpose was to proceed to compensation, and consequently account for the concentration of the payment activities in a few centres. The interdependence of all lines in the accounting document of the balance of payments suggests that there was an interdependence of flows in reality. This hypothesis, still to be verified in facts, leads to the description of a simple and coherent international system in the middle of the $19^{\text {th }}$-century, ensuring a central position to French trade and banking.

\section{AUTEUR}

\section{PATRICK VERLEY}

Professeur à l'Université de Genève 